\title{
Erratum to: Transfer RNA Gene Numbers may not be Completely Responsible for the Codon Usage Bias in Asparagine, Isoleucine, Phenylalanine, and Tyrosine in the High Expression Genes in Bacteria
}

\author{
Siddhartha Sankar Satapathy • Malay Dutta • \\ Alak Kumar Buragohain - Suvendra Kumar Ray
}

Published online: 16 November 2012

(c) Springer Science+Business Media New York 2012

\section{Erratum to: J Mol Evol (2012) 75:34-42 \\ DOI 10.1007/s00239-012-9524-1}

We regret to inform that the data presented in our published article (Satapathy et al. 2012) for the whole genome with respect to amino acid usage and NNC/NNU ratio is erroneous for the two bacteria Pseudomonas aeruginosa and Streptomyces coelicolor in Tables 1 and 2 of the article. The correct data under the "Whole genome" heading for the two bacteria are as follows:

In Table 1

(i) The correct "Whole genome" data for the bacteria P. aeruginosa are 1859569, 3.56, 2.64, 4.17, 2.54 .

(ii) The correct "Whole genome" data for the bacteria S. coelicolor are 2560775, 2.65, 1.70, 2.86, 2.05 .

In view of the above changes in Table 1 , the amino acid usage data of these two bacteria given in the Supplementary Table 1 is no longer required and hence to be ignored.

In Table 2

(i) The correct "Whole genome" data for the bacteria P. aeruginosa are 19.62, 6.06, 13.25, 3.82.

The online version of the original article can be found under doi:10.1007/s00239-012-9524-1.

S. S. Satapathy · M. Dutta

Departments of Computer Science and Engineering,

Tezpur University, Tezpur 784 028, Assam, India

A. K. Buragohain · S. K. Ray $(\square)$

Departments of Molecular Biology and Biotechnology,

Tezpur University, Tezpur 784 028, Assam, India

e-mail: suven@tezu.ernet.in (ii) The correct "Whole genome" data for the bacteria S. coelicolor are 61.99, 23.83, 46.16, 20.5 .

In view of the above corrections in Table 1 , what has been stated in the first paragraph of the result section in the article about the amino acid composition difference between the whole genomes and high expression genes in these two bacteria is no longer valid and hence to be ignored.

In view of the above corrections in Table 2, the NNC/ NNU results for the two bacteria are in agreement with the earlier observation of Sharp et al. (2005). Hence what has been stated in the third paragraph in the discussion section of the article is no longer valid and hence to be ignored.

The changes in Tables 1 and 2 have no effect on the main findings of the analyses presented in the article. There is no change in the Title, Abstract, and Reference sections of the article.

Corrected tables with the relevant changes in the footnote are given below. 
Table 1 Usage (in \%) of phenylalanine (Phe), asparagine (Asn), isoleucine (Ile) and tyrosine (Tyr) amino acids

\begin{tabular}{|c|c|c|c|c|c|c|c|c|c|c|c|c|c|c|}
\hline \multirow[t]{2}{*}{ S. no. } & \multirow[t]{2}{*}{ Strain } & \multirow[t]{2}{*}{ Group } & \multirow[t]{2}{*}{ Genome size } & \multirow[t]{2}{*}{$\mathrm{GC}(\%)$} & \multicolumn{5}{|c|}{ Top $100 \mathrm{HE}$ genes } & \multicolumn{5}{|c|}{ Whole genome } \\
\hline & & & & & Total & Phe & Asn & Ile & Tyr & Total & Phe & Asn & Ile & Tyr \\
\hline 1 & S. aureus & Firmicutes & 2903636 & 32.84 & 33828 & 3.50 & 4.70 & 7.42 & 2.82 & 801462 & 4.48 & 5.69 & 8.59 & 3.93 \\
\hline 2 & S. mutans & Firmicutes & 2030921 & 36.83 & 39508 & 3.60 & 5.06 & 6.85 & 3.26 & 579702 & 4.76 & 4.85 & 7.74 & 3.85 \\
\hline 3 & L. monocytogenes & Firmicutes & 2944528 & 37.98 & 31671 & 3.72 & 4.58 & 6.96 & 3.2 & 870878 & 4.53 & 4.62 & 7.84 & 3.45 \\
\hline 4 & H. influenzae & $\gamma$ Proteobacteria & 1830069 & 38.15 & 25071 & 4.38 & 5.23 & 6.75 & 3.28 & 521077 & 4.48 & 4.88 & 7.11 & 3.14 \\
\hline 5 & B. subtilis & Firmicutes & 4214630 & 43.52 & 32519 & 3.65 & 4.06 & 6.45 & 3.02 & 1228408 & 4.50 & 3.95 & 7.37 & 3.49 \\
\hline 6 & L. plantarum & Firmicutes & 3348625 & 44.42 & 32119 & 3.63 & 4.58 & 6.54 & 3.50 & 920243 & 3.97 & 4.41 & 6.56 & 3.52 \\
\hline 7 & E. coli & $\gamma$ Proteobacteria & 4639675 & 50.00 & 22219 & 3.34 & 3.94 & 5.99 & 2.58 & 1313473 & 3.90 & 3.89 & 6.01 & 2.83 \\
\hline 8 & N. europaea & $\beta$ Proteobacteria & 2812094 & 50.72 & 22536 & 4.05 & 3.70 & 6.17 & 2.95 & 800071 & 3.92 & 3.58 & 6.39 & 2.84 \\
\hline 9 & P. syringae & $\gamma$ Proteobacteria & 6538260 & 58.34 & 26326 & 3.18 & 3.26 & 5.10 & 2.87 & 1814263 & 3.63 & 3.19 & 4.98 & 2.55 \\
\hline 10 & B. longum & Actinobacteria & 2260266 & 60.13 & 30848 & 3.55 & 3.90 & 6.04 & 2.91 & 640513 & 3.41 & 3.43 & 5.37 & 2.68 \\
\hline 11 & D. vulgaris & $\delta$ Proteobacteria & 3773159 & 63.28 & 26270 & 3.49 & 3.03 & 5.09 & 2.55 & 1020841 & 3.59 & 2.41 & 4.45 & 2.27 \\
\hline 12 & B. japonicum & $\alpha$ Proteobacteria & 9105828 & 64.06 & 23090 & 3.55 & 3.61 & 5.29 & 2.70 & 2634346 & 3.73 & 2.76 & 5.26 & 2.21 \\
\hline 13 & R. palustris & $\alpha$ Proteobacteria & 5467640 & 65.03 & 30075 & 3.91 & 3.96 & 5.46 & 3.20 & 1580833 & 3.64 & 2.58 & 5.26 & 2.22 \\
\hline 14 & P. aeruginosa & $\gamma$ Proteobacteria & 6264404 & 66.56 & 21744 & 3.59 & 3.68 & 5.05 & 2.72 & 1859569 & 3.56 & 2.64 & 4.17 & 2.54 \\
\hline 15 & $R$. sphaeroides & $\alpha$ Proteobacteria & 4603060 & 68.79 & 23416 & 3.74 & 3.25 & 5.56 & 2.72 & 943868 & 3.49 & 2.02 & 4.52 & 1.91 \\
\hline 16 & T. thermophilus & Deinococcus-Thermus & 2116056 & 69.50 & 23854 & 3.45 & 2.84 & 4.87 & 3.11 & 589163 & 3.77 & 1.55 & 2.67 & 2.87 \\
\hline 17 & S. coelicolor & Actinobacteria & 9054847 & 72.00 & 27997 & 2.68 & 2.61 & 4.02 & 2.33 & 2560775 & 2.65 & 1.70 & 2.86 & 2.05 \\
\hline
\end{tabular}

Table presents total number of codons and percentage of codons encoding four amino acids, phenylalanine (Phe), asparagine (Asn), isoleucine (Ile) and tyrosine (Tyr) in top 100 high expression (HE) genes as well as in the whole genome of seventeen bacteria (Bacillus subtilis, Bifidobacterium longum, Bradyrhizobium japonicum, Desulfovibrio vulgarisHildenborough, Escherichia coli, Haemophilus influenzae, Lactobacillus plantarum, Listeria monocytogenes, Nitrosomonas europaea, Pseudomonas aeruginosa, Pseudomonas syringae, Rhodobacter sphaeroides, Rhodopseudomonas palustris, Staphylococcus aureus, Streptococcus mutans, Streptomyces coelicolor, Thermus thermophilus)

Table 2 Transfer RNA gene number and synonymous codon usage in phenylalanine (Phe), asparagine (Asn), isoleucine (Ile) and tyrosine (Tyr) amino acids

\begin{tabular}{|c|c|c|c|c|c|c|c|c|c|c|c|c|c|}
\hline \multirow{2}{*}{$\begin{array}{l}\text { S. } \\
\text { no. }\end{array}$} & \multirow[t]{2}{*}{ Name } & \multicolumn{4}{|c|}{ tRNA Gene number } & \multicolumn{4}{|c|}{ Top100 HE genes ${ }^{b}$} & \multicolumn{4}{|c|}{ Whole genome ${ }^{\mathrm{b}}$} \\
\hline & & $\begin{array}{l}\text { Phe } \\
\text { GAA }\end{array}$ & $\begin{array}{l}\text { Asn } \\
\text { GTT }\end{array}$ & $\begin{array}{l}\text { Ile } \\
\text { GAT }\end{array}$ & $\begin{array}{l}\text { Tyr } \\
\text { GTA }\end{array}$ & $\begin{array}{l}\text { Phe } \\
\text { UUC/ } \\
\text { UUU }\end{array}$ & $\begin{array}{l}\text { Asn } \\
\text { AACl } \\
\text { AAU }\end{array}$ & $\begin{array}{l}\text { Ile } \\
\text { AUC/ } \\
\text { AUU }\end{array}$ & $\begin{array}{l}\text { Tyr } \\
\text { UAC/ } \\
\text { UAU }\end{array}$ & $\begin{array}{l}\text { Phe } \\
\text { UUC/ } \\
\text { UUU }\end{array}$ & $\begin{array}{l}\text { Asn } \\
\text { AAC/ } \\
\text { AAU }\end{array}$ & $\begin{array}{l}\text { Ile } \\
\text { AUC/ } \\
\text { AUU }\end{array}$ & $\begin{array}{l}\text { Tyr } \\
\text { UAC/ } \\
\text { UAU }\end{array}$ \\
\hline 1 & S. aureus & 2 & 3 & 2 & 2 & 1.02 & 0.75 & 0.51 & 0.51 & 0.37 & 0.31 & 0.28 & 0.28 \\
\hline 2 & S. mutans & 2 & 2 & 2 & 2 & 0.38 & 0.33 & 0.35 & 0.35 & 0.25 & 0.24 & 0.30 & 0.27 \\
\hline 3 & $\begin{array}{l}\text { L. } \\
\quad \text { monocytogenes }\end{array}$ & 2 & 4 & 3 & 2 & 1.11 & 1.18 & 0.67 & 0.79 & 0.47 & 0.45 & 0.36 & 0.46 \\
\hline 4 & H. influenzae & 1 & 2 & 3 & 1 & 0.45 & 0.36 & 0.36 & 0.33 & 0.38 & 0.33 & 0.28 & 0.28 \\
\hline 5 & B. subtilis & 3 & 4 & 3 & 2 & 0.78 & 1.36 & 1.06 & 0.81 & 0.46 & 0.77 & 0.73 & 0.53 \\
\hline 6 & L. plantarum & 2 & 5 & 3 & 2 & 0.59 & 0.72 & 0.58 & 0.73 & 0.53 & 0.66 & 0.52 & 0.63 \\
\hline 7 & E. coli & 2 & 4 & 3 & 3 & 2.42 & 4.54 & 1.99 & 1.88 & 0.74 & 1.24 & 0.83 & 0.76 \\
\hline 8 & N. europaea & 1 & 1 & 1 & 1 & 0.70 & 0.72 & 0.74 & 0.62 & 0.98 & 0.68 & 1.49 & 0.68 \\
\hline 9 & P. syringae & 1 & 2 & 5 & 1 & 1.38 & 3.03 & 2.30 & 0.94 & 1.81 & 2.36 & 2.25 & 1.80 \\
\hline 10 & B. longum & 1 & 3 & 1 & 1 & 25.05 & 9.95 & 4.85 & 8.07 & 6.89 & 2.81 & 2.81 & 2.24 \\
\hline 11 & D. vulgaris & 1 & 2 & 5 & 1 & 12.10 & 5.91 & 7.84 & 4.32 & 7.04 & 4.37 & 7.49 & 2.43 \\
\hline 12 & B. japonicum & 2 & 2 & 1 & 1 & 2.55 & 1.77 & 3.12 & 1.51 & 4.70 & 2.21 & 7.16 & 1.15 \\
\hline 13 & R. palustris & 1 & 1 & 2 & 1 & 12.21 & 4.54 & 10.27 & 2.46 & 6.24 & 2.40 & 8.19 & 1.33 \\
\hline 14 & P. aeruginosa & 1 & 2 & 4 & 1 & 40.05 & 10.13 & 11.66 & 5.09 & 19.62 & 6.06 & 13.25 & 3.82 \\
\hline 15 & $R$. sphaeroides & 1 & 1 & 3 & $0^{\mathrm{a}}$ & 37.09 & 11.06 & 31.50 & 1.86 & 11.96 & 3.63 & 18.90 & 1.12 \\
\hline 16 & T. thermophilus & 1 & 1 & 1 & 1 & 5.65 & 134.40 & 7.30 & 56.15 & 4.68 & 40.06 & 9.30 & 23.38 \\
\hline 17 & S. coelicolor & 1 & 2 & 1 & 1 & 73.90 & 59.83 & 64.41 & 35.22 & 61.99 & 23.83 & 46.16 & 20.5 \\
\hline
\end{tabular}

${ }^{a}$ No tRNA gene according the Genomic tRNA database (http://gtrnadb.ucsc.edu)

b The ratio between abundance values of the two synonymous codons of an amino acid in the high expression (HE) genes and in the whole genome 


\section{References}

Satapathy SS, Dutta M, Buragohain AK, Ray SK (2012) Transfer RNA gene numbers may not be completely responsible for the codon usage bias in asparagine, isoleucine, phenylalanine, and tyrosine in the high expression genes in bacteria. J Mol Evol 75:34-42

Sharp PM, Bailes E, Grocock RJ, Peden JF, Sockett RE (2005)

Variation in the strength of selected codon usage bias among bacteria. Nucleic Acids Res 33:1141-1153 